\title{
Transient Receptor Potential Channels in Chemotherapy-Induced Neuropathy
}

\author{
Romina Nassini ${ }^{*}, 1$, Silvia Benemei ${ }^{1}$, Camilla Fusi ${ }^{1}$, Gabriela Trevisan ${ }^{1,2}$ and Serena Materazzi ${ }^{1}$ \\ ${ }^{1}$ Department of Preclinical and Clinical Pharmacology, University of Florence, Florence, Italy; ${ }^{2}$ Department of Chem- \\ istry, Federal University of Santa Maria, Santa Maria, Brazil
}

\begin{abstract}
Chemotherapy-Induced Peripheral Neuropathy (CIPN) is a common dose-limiting side effect of many chemotherapeutic drugs, including platinum-based compounds (e.g., cisplatin and oxaliplatin), taxanes (e.g., paclitaxel), vinca alkaloids (e.g., vincristine), and the first-in-class proteasome inhibitor, bortezomib. Among the various sensory symptoms of CIPN, paresthesia, dysesthesia, spontaneous pain, and mechanical and thermal hypersensitivity are prominent. Inflammation, oxidative stress, loss of intraepidermal nerve fibers, modifications of mitochondria, and various ion channels alterations are part of the several mechanisms contributing to CIPN. Because attempts to mitigate chemotherapeutic-induced acute neuronal hyperexcitability and the subsequent peripheral neuropathy have yielded unsatisfactory results, a more in-depth understanding of the mechanism(s) responsible for the neurotoxic action of anticancer drugs is required.

Some members of the transient receptor potential (TRP) family of channels, as the TRPV1 and TRPV4 (vanilloid), TRPA1 (ankyrin) and TRPM8 (melastatin) are expressed on the plasma membrane of primary sensory neurons (nociceptors), where they are activated by an unprecedented series of physical and chemical stimuli. There is evidence that TRPV1, TRPV4, TRPA1 and TRPM8 are prominent contributors of mechanical and thermal hypersensitivity in models of CIPN. In particular, in vitro and in vivo studies have pointed out the unique role of TRPA1 and oxidative stress in the mechanism responsible for cold and mechanical hyperalgesia in rodent models of CIPN.
\end{abstract}

Keywords: Chemotherapy-Induced Peripheral Neuropathy, Transient Receptor Potential Channels (TRP), Primary sensory neurons, Anticancer drugs, Oxidative stress.

\section{INTRODUCTION}

Peripheral neuropathy is an adverse effect common to various chemotherapeutic agents, including vincristine, paclitaxel, oxaliplatin, cisplatin, bortezomib, and thalidomide [1]. Chemotherapy-induced peripheral neuropathy (CIPN) represents a dose-limiting adverse reaction, which negatively affects the quality of life of a relevant part of treated patients and their therapeutic management. The incidence of CIPN is lower in patients treated with a single agent (3-7\%), but can rise up to $38 \%$ in patients treated with combination regimens [2]. The occurrence and severity of CIPN depend on many factors, including dose intensity, treatment duration, cumulative dose, prior or concurrent treatment with other neurotoxic drugs, and co-existing conditions which give an independent risk of neuropathy, such as diabetes and alcohol abuse.

The platinum-based anticancer drugs cisplatin, carboplatin, and oxaliplatin are successfully used for the treatment of lung, colorectal, ovarian, breast, head and neck, bladder, and testicular cancers. Cisplatin neurotoxicity is predominantly characterized by sensory neuropathy, which principally produces pain and paresthesia in the extremities.

*Address correspondence to this author at the Department of Preclinical and Clinical Pharmacology, University of Florence, Viale Pieraccini 6, 50139 Florence, Italy; Tel: +39 055427 1417; Fax: +39 055427 1280;

E-mail: romina.nassini@unifi.it

1876-3863/13
This sensory neuropathy may have a delayed onset, appearing weeks after treatment initiation and, in advanced stages, it may progress to severe neuropathic pain and sensory ataxia. The third generation platinum drug oxaliplatin, which is the most active for the treatment of colorectal cancer [3], shows a dramatic reduction in renal toxicity and ototoxicity $[4,5]$, but exhibits a unique neurotoxic profile. Oxaliplatin induces an acute painful neuropathy which appears soon after administration [6]. Patients complain about paresthesia, more often localized periorally and/or to the extremities, and severe cold hypersensitivity. In about $90 \%$ of patients, exposure to cold triggers or enhances an acute, transient syndrome characterized by cramps, paresthesia, and dysesthesia. A chronic peripheral neuropathy resembling that linked to cisplatin often develops after multiple treatment cycles with oxaliplatin.

Paclitaxel is a microtubule-targeting agent labeled for the treatment of a wide variety of solid neoplasms currently under investigation to assess its efficacy to treat additional malignant tumors. Paclitaxel-induced neurotoxicity typically presents as a sensory neuropathy with the most common complaints being numbness, tingling, and burning pain. More pronounced symptoms are tingling and allodynia which typically occur in a "glove and stocking" distribution. Sensory symptoms usually start symmetrically in the feet, but also appear simultaneously in both hands and feet [7]. Many cases resolve briefly after paclitaxel discontinuation, 
but the sensory abnormalities and pain can be long-lasting [8].

Among other anticancer drugs, the vinca alkaloid vincristine is a widely used antineoplastic agent administered alone or in combination with other drugs in the treatment of many tumor types [9, 10]. Bortezomib is a modified dipeptidyl boronic acid authorized for the treatment of multiple myeloma and mantle cell lymphoma [11-13]. Recently, thalidomide has received a great deal of attention due to its remarkable therapeutic efficacy in the treatment of multiple myeloma [14]. Vincristine, bortezomib, and thalidomide can also cause CIPN.

Various mechanisms have been suggested to play a role in the development of CIPN and have been explored in rodent models. Different mitochondrial pathways, including regulation of intracellular calcium [15], generation of reactive oxygen species (ROS) [16], and apoptotic signaling [17], have been proposed to contribute to the development of CIPN [18]. Indeed, paclitaxel-evoked painful peripheral neuropathy is associated with increased swollen and vacuolated axonal mitochondria [19]. Moreover, paclitaxel appears to gate the multi-molecular complex containing the voltagedependent anion channel, defined as mitochondrial permeability transition pore (mPTP) [19], thus causing a toxic calcium release from the mitochondria [20]. In accordance with this observation, calcium chelating agents are able to reverse paclitaxel-evoked pain [21], and acetyl-1-carnitine, which prevents mPTP opening [22], reduces the development of paclitaxel-induced neuropathic pain [23]. Administration of bortezomib leads to intracytoplasmatic vacuolization in dorsal root ganglia (DRG) satellite cells, probably due to mitochondrial and endoplasmic reticulum enlargement [24]. All these intracellular modifications are probably related to the ability of bortezomib to activate mitochondrial-based apoptotic pathways, including activation of caspases [25] and dysregulation of calcium homeostasis [26]. Inhibitors of the mitochondrial electron transport chain (mETC) attenuate mechanical hyperalgesia in both CIPN models and after tumor necrosis factor- $\alpha$ (TNF- $\alpha$ ) treatment [17]. The relevant role of $\mathrm{mETC}$ in peripheral pain mechanisms is further corroborated by the effect of inhibitors of adenosine triphosphate (ATP) synthesis to attenuate neuropathic pain [17]. Moreover, it has been demonstrated that the antioxidant agent $\alpha$-lipoic acid, by regulating essential mitochondrial proteins with antioxidant and chaperone properties, exerts neuroprotective effects against chemotherapy-induced neurotoxicity in sensory neurons [27]. Finally, significant changes in the expression of various genes, including those controlling mitochondrial dysfunction associated with vincristine- and bortezomib-evoked peripheral neuropathy, have been demonstrated in humans [25].

Impaired mitochondrial calcium uptake, or increased leakage of mitochondrial calcium, could exaggerate calcium signals and, eventually, calcium-dependent processes which participate in the neuropathy mechanism. For instance, it has been observed that administration of vincristine and paclitaxel, by raising neuronal calcium levels in the nerves, induces mitochondrial changes, associated with neuronal hyperexcitability $[28,29]$. Accordingly, drugs, which reduce intracellular calcium levels are able to reverse the negative effects of altered mitochondrial calcium regulation and neu- ropathic pain [20,21]. Furthermore, paclitaxel- and vincristine-evoked neuropathic pain is reduced by both the T-type channel calcium blocker, ethosuximide, and the $\alpha 2 \delta$ calcium channel subunit antagonist, gabapentin [30, 31]. In addition, paclitaxel has been reported to increase the expression level of $\alpha 2 \delta-1$ mRNA in the dorsal spinal cord [30, 32]. Accordingly, it has been proposed that $\alpha 2 \delta-1$ subunit in the spinal dorsal horn and DRG is a main site where gabapentin inhibits paclitaxel-induced allodynia [33]. Thus, different lines of evidence indicate that dysregulation of intracellular calcium levels represents an additional factor contributing to the pathogenesis of CIPN.

A number of studies suggest a role of sodium channels in CIPN. Exposure of DRG neurons to oxaliplatin increases sodium currents, which are antagonized by the sodium channel blocker carbamazepine [34]. The oxaliplatin metabolite oxalate probably alters the functional properties of voltagegated sodium channels, resulting in a prolonged open state of the channels and, finally, in the hyperexcitability of sensory neurons [35]. Further, oxaliplatin administration has been described to slow sodium channel inactivation kinetics [1, 34]. A change in sodium channel properties may predispose to ectopic activity, leading to paresthesia and fasciculations [36]. Cold exposure affects sodium channel kinetics [37] and, accordingly, sodium channel dysfunction is aggravated by cold temperatures [38]. Cold hypersensitivity is a typical feature observed in acute oxaliplatin-induced neurotoxicity. It has been shown that acute modulation of sodium channels influences the severity of oxaliplatin-induced neurotoxicity $[39,40]$. The involvement of sodium channels is also reported in paclitaxel-induced neuropathic pain, where low doses of tetrodotoxin result able to prevent pain induced by taxane [41]. In contrast with these previous findings, antisense oligodeoxynucleotides targeting the $\mathrm{Na}_{\mathrm{v}} 1.8$ channel does not seem to interfere with vincristine-induced neuropathic pain [42].

An important function of inflammatory mediators has been described in models of CIPN [29, 43]. A recent study demonstrated a correlation between the increase in interleukin 6 (IL-6) and the appearance of bortezomibinduced neuropathic pain [44]. Further, the administration of the prostaglandin $\mathrm{E}_{1}\left(\mathrm{PGE}_{1}\right)$ analog, limaprost, attenuated mechanical allodynia induced by paclitaxel and oxaliplatin (but not by vincristine) [32]. An increase in skin Langerhans cells (LC) has been associated with the development of pain in vincristine- and paclitaxel-evoked neuropathy [21]. LC cells may contribute to pain development by different mechanisms including release of nitric oxide (NO) [45], proinflammatory cytokines [46], and neurotrophic factors [47], that in turn cause spontaneous neuronal discharge, nociceptor sensitization, and mechano-hypersensitivity. It has also been demonstrated that paclitaxel-induced neuropathic pain is associated with TNF- $\alpha$ and IL- $1 \beta$ induction in lumbar DRGs [48]. Glial cell inhibitors attenuate paclitaxel- and vincristine-induced neuropathic pain $[49,50]$, supporting a role for activated glial cells in this condition.

In vincristine- and paclitaxel-evoked neuropathy [21], and more recently in oxaliplatin-induced neuropathy [51], a loss of intraepidermal nerve fibers in the plantar hind paw skin region of the sensory neuron peripheral terminal arbors, similar to that documented in other neuropathic pain syn- 
dromes, has been shown. Neuropathy also seems to be characterized by a loss of the cutaneous $\mathrm{A} \delta$ and $\mathrm{C}$ fibers (cooland warm-specific) [52] and of $A \delta$ cool-specific fibers, which seem to contribute to cold allodynia [53]. Oxidative stress has been repeatedly proposed to play a central role in the mechanism of CIPN. The effect of antioxidants, including acetyl-1-carnitine, $\alpha$-lipoic acid, and vitamin $\mathrm{C}$, which seem to partially reverse the hyperalgesia, represents indirect proof of the role of oxidative stress in oxaliplatin-induced neuropathy [54, 55]. Recently, administration of the free radicals scavenger, phenyl N-tert-butylnitrone, has been shown to reduce mechanical allodynia in paclitaxel-induced neuropathic pain in rats [56]. Moreover, it has been demonstrated that bortezomib increases ROS in DRG neurons [57], and that vitamin $\mathrm{C}$ or $\mathrm{N}$-acetyl-1-cysteine administration alleviates the cytotoxicity in Schwann cells, but not in myeloma cells treated with bortezomib [58], thus suggesting that the antioxidant action may selectively afford protection against neurodegeneration without modification of the antineoplastic activity of the chemotherapeutic agent [58].

Recent evidence also supports the role of other biological effectors in CIPN. For instance, paclitaxel-induced peripheral neuropathy is characterized by the activation of calciumactivated proteases, such as calpains and caspases [17, 59], or mitogen activated protein kinase (MAPK). Furthermore, prolonged exposure to oxaliplatin induces early activation of p38 and extracellular signal-regulated kinase 1/2 (ERK1/2) MAPKs in DRG neurons, eventually provoking neuronal apoptosis. Contrasting data have been reported on the role of neuropeptides, such as calcitonin gene related peptide (CGRP) or substance P (SP) [60-62]. The role of NO has also been evaluated, and there is indication that NO contributes to vincristine- and oxaliplatin-induced neuropathy. Finally, a number of other mediators or effector mechanisms have been implicated in the genesis of CIPN, including $N$ methyl-D-aspartate (NMDA) and 5-hydroxytryptamine $(5 \mathrm{HT})$ receptors, potassium channels, protein kinase $\mathrm{C}$ (PKC) or 1-serine (see for review [63]).

Recently, in addition to classical calcium or sodium channels, remarkable interest has been paid to a possible role in CIPN of the additional channels preferentially located in sensory neuronal membranes. In particular, research on transient receptor potential (TRP) channels seems to represent a promising area of investigation, as emerging and compelling data have shown the contribution of several members of this channel family to the mechanism of CIPN.

\section{TRP CHANNELS IN PRIMARY SENSORY NEU- RONS: ROLE IN PAIN TRASMISSION}

Neuropathic pain is characterized by hypersensitivity to mechanical, thermal and/or chemical stimuli, elicited by exogenous, or endogenous causes, including trauma, neurotoxins, infections, heredity, immunological and metabolic diseases, and other conditions. A variety of molecular mechanisms have been advocated as underlying pathways contributing to pain hypersensitivity. In particular, activation and sensitization of nociceptors have been considered as an initial mechanism that eventually results in neuronal hypersensitivity. The subpopulation of primary sensory neurons encompasses highly heterogeneous subgroups of neurons. In addition to morphological, electrophysiological, and func- tional criteria, primary sensory neurons may be distinguished according to the expression of neuropeptides, namely the tachykinins SP and the neurokinin A, and CGRP, which upon release from peripheral nerve endings mediate neurogenic inflammation $[64,65]$. Indeed, the first description of the ability of a subset of sensory neurons to orchestrate an early inflammatory response, mainly represented by arteriolar vasodilatation, was reported by Bayliss [66], and subsequently by Sir Thomas Lewis [67], who defined in detail the dual "nocifensor" role of this type of neuron.

Nociceptors that release neuropeptides, thus contributing to neurogenic inflammation, are known to express some TRP channels, which, among other features, are sensitive to changes in temperature and therefore defined also as thermoTRPs [68]. About 15 years after the cloning of the vanilloid 1 channel (TRPV1, the so called 'capsaicin receptor'), additional members of the TRP family have been found to be expressed by nociceptors [69]. These include the vanilloid 2 (TRPV2), 3 (TRPV3), and 4 (TRPV4) channels, the TRPM8 (the 'menthol receptor'), and the ankyrin 1 (TRPA1) channels. These channels are transducers of an unprecedented series of chemical, thermal, and mechanical stimuli that are usually known to induce pain. Although the hallmark of TRP channels is their "polymodality", TRPV1, TRPV3, TRPM8, and TRPA1 have also been recognized as chemoreceptors, rather selectively responsive to capsaicin, camphor [70], menthol [71, 72], and mustard oil [73], respectively. The reader is referred to other reviews for detailed descriptions of the specific thermal, mechanical, and chemical sensitivity of TRP channels expressed by nociceptors $[65,68]$.

\section{THE ROLE OF TRP CHANNELS IN CHEMOTHERA- PY-INDUCED PERIPHERAL NEUROPATHY}

Pharmacological and genetic studies using animal models of CIPN induced by various chemotherapeutic agents indicate that the mechanisms underlying mechanical and thermal hyperalgesia are multiple. Notwithstanding, recent evidence has emphasized a primary role for members of the TRP family, in particular TRPV1, TRPV4, TRPA1, and TRPM8, in the mechanical and thermal hypersensitivity evoked by chemotherapeutic agents in rodents [62, 74, 75].

\section{TRPV1}

The invertebrate relatives of TRPV1 are essential to sensory transduction (phototransduction, thermosensation, mechanosensation, osmosensation) [76], while in mammals TRPV1 seems to contribute to hypersensitivity to thermal, chemical, and mechanical stimuli associated with peripheral inflammation and neuronal damage. A specific feature of TRPV1 relies on its ability, following intense and prolonged activation, to induce neuronal desensitization $[77,78]$. TRPV1 is activated by noxious heat $\left(43-52^{\circ} \mathrm{C}\right)$, and its activation by capsaicin results in heat hypersensitivity [79]. Among various adverse reactions, heat hypersensitivity has been often reported by patients treated with platinum-based anticancer drugs [80]. Thus, the hypothesis that TRPV1 plays a role in such reactions has been advanced. Treatment with cisplatin has been found to produce upregulation of TRPV1 mRNA in cultured DRG neurons [81]. A similar upregulation occurs also after in vivo treatment with cisplatin, although cisplatin-treated mice showed no change in 
the proportion of TRPV1-immunopositive trigeminal ganglia (TG) neurons [82]. TRPV1 upregulation was associated with increased nociceptors responsiveness and contributed to cisplatin-evoked thermal, but not mechanical, hyperalgesia in mice [82]. In addition, acute exposure to oxaliplatin induced TRPV1 sensitization, which may cause neuronal damage [81]. The mechanism through which oxaliplatin/cisplatininduced neuropathy results in TRPV1 sensitization is unclear. However, enhanced TRPV1 protein trafficking, consequent upon mRNA overexpression, to peripheral nerve processes, or channel phosphorylation by different kinases, leading to enhanced TRPV1 sensitivity, have been proposed as a general mechanism contributing to pathological pain states [83].

\section{TRPV4}

TRPV4 channel is a polymodal receptor with a wide expression pattern and a corresponding variety of possible pathophysiological roles [84]. TRPV4 is expressed in different neuronal and non-neuronal cells, including urinary bladder, kidney, vascular endothelium, keratinocytes, cochlear hair cells, and Merkel cells [85-87]. Likewise the TRPV1 channel, TRPV4 activation on TG or DRG neurons [84, 88] causes SP and CGRP release, thus evoking neurogenic inflammation in peripheral tissues [89]. TRPV4 was firstly identified as an osmo-transducer activated by decrease in osmolarity, suggesting a role in the regulation of cell swelling $[84,90]$. Later studies demonstrated that TRPV4 is activated by shear stress [91], innocuous warmth $\left(27-35{ }^{\circ} \mathrm{C}\right)$ [88, 92], low pH, citrate [87], endocannabinoids and arachidonic acid metabolites [92, 93], NO [94], and synthetic selective agonists, such as the phorbol ester $4 \alpha$-phorbol 12,13didecanoate (4 $\alpha$-PDD) [95].

The mechanosensitive nature of TRPV4 and its implication in sensing shear stress suggest a role in flow-sensitive cells, such as vascular endothelial and renal tubular epithelial cells. The mechanism through which TRPV4 is activated by mechanical stress is still under debate. Two transduction pathways have been proposed to regulate TRPV4 activation: the phospholipase (PL) C (PLC)/diacylglycerol (DAG) pathway and the $\mathrm{PLA}_{2}$ /arachidonic acid (AA) pathway [96, 97]. Some evidence suggests that activation of TRPV4 by hypotonicity involves its phosphorylation by the Src family of tyrosine kinase [98]. Although the molecular mechanism of hypotonicity-induced TRPV4 activation should be further investigated, studies addressing the gating mechanism of the channel by cell swelling exclude that it is directly gated by mechanotransduction since it does not respond to membrane stretch [90].

It has been shown that hypotonicity becomes painful to the animals when nociceptive fibers are sensitized by the $\mathrm{PGE}_{2}$, whose levels increase during inflammation or in response to mechanical, chemical, and thermal injury. TRPV4 also plays a crucial role in mechanical hyperalgesia elicited by exposure to inflammatory mediators. Indeed, $\mathrm{PGE}_{2}$ and serotonin can act synergistically through cAMP/protein kinase (PK) A (PKA) and PKC $\varepsilon$ to engage TRPV4 in hyperalgesia to mechanical and osmotic stimuli [99]. In addition, protease-activated receptor $2\left(\mathrm{PAR}_{2}\right)$ agonists may sensitize TRPV4 through the activation of multiple second messenger pathways, such as PKA, PKC, PKD, and PLC $\beta$ [89]. Prote- ases generated during inflammation activate $\mathrm{PAR}_{2}$, thus leading to TRPV4-mediated release of SP and CGRP in the spinal cord and TRPV4-induced mechanical hyperalgesia [100].

Recent evidence has proposed a role for TRPV4 in mechanical allodynia in rodent models of CIPN [101-103]. In different models of painful peripheral neuropathy, mechanical hyperalgesia was markedly reduced by spinal intrathecal administration of oligodeoxynucleotides antisense to TRPV4 [74]. TRPV4 knock-out mice showed reduced mechanical hyperalgesia induced by the anticancer drugs, paclitaxel and vincristine, or in a diabetic model [74]. TRPV4 plays a major role in mechanical hyperalgesia, and also contributes to enhanced nociception to hypo-osmotic stimuli in paclitaxeltreated rats. TRPV4-mediated hypersensitivity by paclitaxel is not attributable to increased mRNA levels, but rather it may be related to a specific interaction with second messenger pathways [101]. Similarly to paclitaxel, treatment with vincristine has been reported to produce mechanical allodynia in rodents through a TRPV4-dependent mechanism [74]. Authors suggest that TRPV4 is not directly activated by these agents, but plays a role in mechanotransduction, as a component of a molecular complex that functions only in presence of inflammation or nerve injury phenomena. This complex pathway results in the activation of a signaling cascade initiated by integrins which, via Src tyrosine kinase, induces membrane insertion and/or activation of the TRPV4 channel in sensory neurons. Tyrosine kinases are known to regulate trafficking of ion channels and receptors. Recent reports demonstrate that Src tyrosine kinases participate in the modulation of TRP channel function [98, 104, 105], and this mechanism could be responsible for TRPV4 sensitization. In paclitaxel-induced peripheral neuropathy TRPV4mediated mechanical hyperalgesia results essentially dependent on integrin/Src tyrosine kinase signaling [101].

Another recent paper demonstrates that paclitaxel may release mast cell tryptase, which activates $\mathrm{PAR}_{2}$ receptors expressed in primary sensory neurons [100]. $\mathrm{PAR}_{2}$ activation and the downstream enzymes, PKA, PKCE, and PLC, cause sensitization of TRPV1, TRPV4, and TRPA1, thereby leading to mechanical allodynia and thermal hyperalgesia. Targeting the signaling pathways of $\mathrm{PAR}_{2}$ seems to effectively attenuate paclitaxel-induced mechanical, heat, or cold hypersensitivity [100]. The contribution of TRPV4 to CIPN, and more in general to models of inflammatory pain, corroborates the hypothesis that TRPV4 plays a role in sensitization of nociceptors and makes it a novel target for the development of an innovative class of analgesics.

\section{TRPA1}

TRPA1, originally cloned from human fetal lung fibroblasts, is a nonspecific calcium-permeable cationic channel expressed in primary sensory neurons of the DRG, TG and vagal ganglia (VG), where it co-localizes with the TRPV1 channel. TRPA 1 is also widely expressed in many cell types, tissues and organs, including hair cells, pancreas, heart, brain, keratinocytes [106], urinary bladder [107], prostate [108], arteries [109], enterochromaffin cells [110], odontoblasts [111], dental pulp [112], synovial fibroblasts [113], airway epithelial [114], and smooth muscle cells [156]. Transient receptor potential ankyrin 1 channel localized to 
non-neuronal airway cells promotes non-neurogenic inflammation.

It has been proposed that TRPA1 functions as a detector of mechanical stimuli and noxious cold $\left(<17{ }^{\circ} \mathrm{C}\right)$, although this hypothesis is still controversial. Altered mechanical thresholds observed in TRPA1 knock-out mice [115] and interaction between TRPA1 N-terminal ankyrin repeat domain and other proteins, such as cadherin [116], suggested that TRPA1 is involved in mechano-sensation. However, other data failed to find any connection between TRPA1 activation and mechanosensation [117]. Similarly, whether or not TRPA1 functions as a sensor of noxious cold remains an unresolved question. Several studies demonstrated that noxious cold activates TRPA1 channels, both directly [118120] and indirectly [121]. However, negative results were obtained in mouse TRPA1 channels heterologously expressed in human embryonic kidney cells [122], and neuronal activation by cold temperatures was found to be similar between wild-type and TRPA1 knock-out mice [123]. In addition, in vivo studies employing two independently produced TRPA1 knock-out mice breeds yield conflicting results, leaving the controversy unsettled [115, 117, 120]. More recently, it has been proposed that noxious cold activates TRPA1 [124], but with less potency than allyl isothiocyanate. Moreover, it has been shown that cold stimuli potentiated TRPA1 activation induced by allyl isothiocyanate [124].

Whereas the role of TRPA1 in mechano- and coldtransduction remains to be clarified, it has been extensively demonstrated that TRPA1 plays a major role in chemosensation. In fact, TRPA1 is activated by a wide range of pungent and irritant compounds [125], including ingredients of various spicy foods, such as allyl isothiocyanate (mustard oil, wasabi and horseradish) [73], allicin and diallyldisulfide (garlic derivatives) [126], cinnamaldehyde (cinnamon), and environmental irritants and industry pollutants, such as acetaldehyde [127], formalin [128], hypochlorite, isocyanates [129], ozone [130], carbon dioxide [131], and acrolein [117], a highly reactive $\alpha, \beta$-unsaturated aldehyde present in tear gas, and cigarette smoke [132]. Moreover, isofluorane [133], nicotine [134], NO donors [135], and cyclophosphamide [117] have been reported to activate TRPA1.

In addition to exogenous compounds, endogenous molecules have been identified as TRPA1 agonists. Compelling evidence indicates that TRPA1 is gated by an unprecedented series of endogenous agents generated at sites of inflammation and tissue injury. The product of fatty acid metabolism, 15-deoxy-delta-12,14-prostaglandin $\mathrm{J} 2$ (15d-PGJ $\mathrm{d}_{2}$, which is synthesized by cyclooxygenases after an initial inflammatory signal, is a potent activator of TRPA1 [136, 137]. The cyclopentenone isoprostane 8-iso-PGA2 is also capable of targeting TRPA1 [137]. Oxidative decomposition of polyunsaturated fatty acids, such as linoleic and arachidonic acid, leads to the formation of a host of reactive carbonyl species that may target TRPA1. These products include $\alpha, \beta$ unsaturated aldehydes acrolein, 4-hydroxy-2-nonenal (HNE) [138], and 4-oxononenal [139]. Moreover, reactive nitrogen species (RNS) such as peroxynitrite and nitrooleic acid [130] and ROS, such as oxygen peroxide and hydrogen peroxide [140], target TRPA1. Thus, oxidative stress produced by neutrophilic and macrophagic activation at sites of inflam- mation generates molecules that activate TRPA1 leading to pain and neurogenic inflammation. These findings suggest that TRPA1 plays a key role in sensing tissue damage and nociceptive signaling. TRPA1 channel can be gated by distinct mechanisms. Most of TRPA1 activators are characterized by the presence of a highly reactive electrophilic group that, via a Michael-addition reaction, form covalent bonds with nucleophilic groups, such as cysteine and lysine residues located in the N-terminal cytoplasmic domain of the channel, hence inducing modifications of TRPA1 N-terminal that lead to dilation of the channel permeation pore [141, 142]. As in the case of electrophilic agonists, HNE provokes TRPA1 gating by covalent modification of cysteine and lysine residues located within the N-terminal cytoplasmic domain of the channel [138]. Unlike these molecular species, it has been reported that $\mathrm{H}_{2} \mathrm{O}_{2}$ activates TRPA1 via disulfide bond formation induced by oxidation [143].

In addition to direct channel activation, different inflammatory agents that target $\mathrm{G}$ protein coupled receptors (e.g., bradykinin) and tyrosine kinases receptors (e.g., nerve growth factor) can indirectly sensitize TRPA1 by activating PKA or PKC [125]. Similar mechanisms of TRPA1 sensitization have been reported to occur by $\mathrm{PAR}_{2}$ agonists, probably by activation of PLC, which releases the inhibition of TRPA1 from plasma membrane $\mathrm{PIP}_{2}$ [144]. This evidence suggests that TRPA1 functions as an integrator of different inflammatory mediators, in turn leading to amplification of inflammatory and nociceptive signals.

Patients treated with several anticancer drugs develop hypersensitivity to cold stimuli, thus suggesting the involvement of TRPA1 in this adverse reaction. In addition, induction of oxidative stress is a general mechanism that may contribute to the antineoplastic effect of several chemotherapeutic agents [145], and the TRPA1 channel is a sensor of oxidative stress byproducts [140]. Thus, due to its localization on nociceptive sensory neurons, and being a major thermal and oxidative stress target, the TRPA1 receptor seems to be perfectly suited to contribute to symptoms of CIPN.

Recently, our research group has disclosed the role of TRPA1 in models of CIPN [103, 146]. By both genetic and pharmacological approaches, we showed that TRPA1 entirely mediates mechanical and cold hypersensitivity induced by oxaliplatin and cisplatin [146] in mice and rats. We confirmed [103] that TRPV4 mediates part of the mechanical hyperalgesia induced by paclitaxel [74], and we showed that

TRPV4-resistant mechanical hyperalgesia was exclusively mediated by TRPA1 [103]. We also discovered that paclitaxel-induced cold allodynia was completely due to TRPA1 activation [103]. One final common pathway activated by the otherwise chemically heterogeneous group of molecules, such as chemotherapeutic agents, is the production of oxidative stress in different tissues and cells [147, 148] and, through this effect they can potentially activate and/or sensitize the TRPA1 channel. Our recent works [103, 146], however, indicated that oxaliplatin and paclitaxel do not directly gate TRPA 1 , as they do not cause any calcium response in primary culture of mouse or rat DRG neurons. However, Chinese hamster ovary (CHO) cells transfected with the mouse TRPA1 channel respond, with a glutathionesensitive intracellular calcium mobilization, upon challenge 
with oxaliplatin, whereas untransfected $\mathrm{CHO}$ cells do not. Thus, we hypothesized that calcium response by oxaliplatin requires two conditions. The first is that the cell expresses TRPA1, and the second is that the cell may generate sufficient levels of oxidative stress. It is possible that neurons do not produce sufficient oxidative stress to activate TRPA1, whereas $\mathrm{CHO}$ cells possess the metabolic and enzymatic repertoire to produce high enough ROS levels which, when the cells express the recombinant TRPA1, are sufficient to gate the channel. In the case of sensory neurons neighboring cells to nerve terminals may release oxidative stress byproducts generated by paclitaxel, hence gating TRPA1. Paclitaxel seems to utilize the same pathway given that the TRPA1dependent CGRP release evoked by the drug was completely abated in the presence of glutathione [103]. Similarly to what has been found under inflammatory circumstances [149], platinum-based drugs also increase TRPA1 expression in DRG [82]. However, in this study, appropriate functional experiments that could corroborate the intriguing molecular data were not performed.

Moreover, in a rat model of diabetes, paclitaxel significantly enhanced cold hyperalgesia in comparison to normoglycemic paclitaxel-treated control animals [149]. These effects were prevented by the ROS scavenger, N-acetylcysteine, and by the selective TRPA1 antagonist, HC030031 [128]. In diabetic and control rats, paclitaxel treatment was associated with an accumulation of atypical mitochondria and an increase in mitochondrial ROS production [149]. Paclitaxel potentiation of cold hyperalgesia in diabetes may result from the combination of increased mitochondrial ROS production and poor radical detoxification induced by paclitaxel treatment and increased TRPA1 expression [149].

\section{TRPM8}

TRPM8 is expressed by a non-peptidergic subpopulation of nociceptors and responds to mild and noxious cold $(<25$ $\left.{ }^{\circ} \mathrm{C}\right)$ temperatures. TRPM8, together with TRPA1, seems to mediate hypersensitivity to cold stimuli [71]. An increase in TRPM8 expression occurs in some sensory neurons after nerve injury [150], possibly contributing to enhanced cooling sensation. Similarly, oxaliplatin increased the expression of TRPM8 mRNA in mouse DRG neurons when cold hypersensitivity peaked, suggesting that cold hypersensitivity is, at least partly, due to the increased expression of TRPM8 in primary sensory neurons [151]. In addition, wet-dog shake and jumping behaviors elicited by icilin, a non selective TRPM8 activator, were significantly increased in mice treated with oxaliplatin [151]. Oxaliplatin seems to affect TRPA1 rather than TRPM8, because oxaliplatin-treatment induces sensitization to icilin, which also activates TRPA1 expressing neurons, but not the response to a TRPM8 selective ligand, WS12 [81]. A recent paper reported a possible contribution of TRPM8 expressing fibers to cold hypersensitivity induced by oxaliplatin [152]. Paradoxically, a case report showed the analgesic effect of topical menthol application in CIPN induced by bortezomib [153]. In addition, topical application of menthol was able to significantly reverse CIPN induced by carboplatin, and its prolonged application during chemotherapy appeared to prevent neuropathy worsening [154]. More basic and clinical investigations are clearly required to clarify the role of TRPM8 in CIPN.

\section{CONCLUSION}

CIPN encompasses a large variety of symptoms, but neuropathic pain represents a prominent and dose-limiting manifestation for many patients. Although a series of hypotheses has been reported to explain this painful condition, the mechanism underlying CIPN remains unknown, and the disease remains undertreated, the main undesired consequence being therapy discontinuation. However, over the past two years a series of mechanisms and potential targets through which anticancer drugs may induce peripheral neuropathy emerged.

In particular, recent acquisitions on members of the TRP family, such as TRPV1, TRPV4, TRPA1, and TRPM8, expressed by primary nociceptors, indicate that these channels play a major role in models of CIPN. Emerging data show that oxidative stress produced by chemotherapeutic agents initiates channel gating, and this phenomenon seems to be specifically important for TRPA1 activation and sensitization.

However, several questions regarding the relationship between CIPN and TRP channels remain unanswered. Upregulation of TRP channels has been claimed as a possible mechanism for protracted sensory neuropathy, but this phenomenon has not been always demonstrated. It is highly possible that additional molecular mechanisms are involved in TRP hyperactivity, including altered intracellular transduction pathways and/or epigenetic factors, which have been previously proposed to play a key role in models of inflammatory and neuropathic pain [69, 83, 155]. Thus, further studies are required to identify the upstream and downstream events concerning the primary sensory neurons, which are associated to chemotherapeutic agent-evoked and TRPmediated hypersensitivity, and ultimately to CIPN.

\section{CONFLICT OF INTEREST}

The authors confirm that this article content has no conflicts of interest.

\section{ACKNOWLEDGEMENTS}

Supported by grants from the Istituto Italiano di Tecnologia (Grant SEED), and the Ente Cassa di Risparmio di Firenze.

\section{REFERENCES}

[1] Wolf S, Barton D, Kottschade L, et al. Chemotherapy-induced peripheral neuropathy: prevention and treatment strategies. Eur J Cancer 2008; 44: 1507-15.

[2] Connelly E, Markman M, Kennedy A, et al. Paclitaxel delivered as a 3-hr infusion with cisplatin in patients with gynecologic cancers: unexpected incidence of neurotoxicity. Gynecol Oncol 1996; 62: 166-8.

[3] Andre T, Boni C, Mounedji-Boudiaf L, et al. Oxaliplatin, fluorouracil, and leucovorin as adjuvant treatment for colon cancer. N Engl J Med 2004; 350: 2343-51.

[4] McKeage MJ, Hsu T, Screnci D, et al. Nucleolar damage correlates with neurotoxicity induced by different platinum drugs. Br J Cancer 2001; 85: 1219-25.

[5] Grothey A. Oxaliplatin-safety profile: neurotoxicity. Semin Oncol 2003; 30: 5-13.

[6] Pasetto LM, D'Andrea MR, Rossi E, et al. Oxaliplatin-related neurotoxicity: how and why? Crit Rev Oncol Hematol 2006; 59: 15968. 
[7] Dougherty PM, Cata JP, Cordella JV, et al. Taxol-induced sensory disturbance is characterized by preferential impairment of myelinated fiber function in cancer patients. Pain 2004; 109: 132-42.

[8] Rowinsky EK, Eisenhauer EA, Chaudhry V, et al. Clinical toxicities encountered with paclitaxel (Taxol). Semin Oncol 1993; 20: $1-15$.

[9] Weiss HD, Walker MD,Wiernik PH. Neurotoxicity of commonly used antineoplastic agents (second of two parts). N Engl J Med 1974; 291: 127-33.

[10] Kaplan RS,Wiernik PH. Neurotoxicity of antineoplastic drugs. Semin Oncol 1982; 9: 103-30.

[11] Richardson PG, Barlogie B, Berenson J, et al. A phase 2 study of bortezomib in relapsed, refractory myeloma. N Engl J Med 2003; 348: 2609-17.

[12] San Miguel JF, Schlag R, Khuageva NK, et al. Bortezomib plus melphalan and prednisone for initial treatment of multiple myeloma. N Engl J Med 2008; 359: 906-17.

[13] Frankland-Searby S,Bhaumik SR. The 26S proteasome complex: an attractive target for cancer therapy. Biochim Biophys Acta 2012; 1825: 64-76.

[14] Morgan GJ, Krishnan B, Jenner M, et al. Advances in oral therapy for multiple myeloma. Lancet Oncol 2006; 7: 316-25.

[15] Shishkin V, Potapenko E, Kostyuk E, et al. Role of mitochondria in intracellular calcium signaling in primary and secondary sensory neurones of rats. Cell Calcium 2002; 32: 121-30.

[16] Chung JM. The role of reactive oxygen species (ROS) in persistent pain. Mol Interv 2004; 4: 248-50.

[17] Joseph EK, Levine JD. Caspase signalling in neuropathic and inflammatory pain in the rat. Eur J Neurosci 2004; 20: 2896-902.

[18] Jaggi AS, Singh N. Mechanisms in cancer-chemotherapeutic drugsinduced peripheral neuropathy. Toxicology 2012; 291.

[19] Flatters SJ, Xiao WH,Bennett GJ. Acetyl-L-carnitine prevents and reduces paclitaxel-induced painful peripheral neuropathy. Neurosci Lett 2006; 397: 219-23.

[20] Kidd JF, Pilkington MF, Schell MJ, et al. Paclitaxel affects cytosolic calcium signals by opening the mitochondrial permeability transition pore. J Biol Chem 2002; 277: 6504-10.

[21] Siau C, Bennett GJ. Dysregulation of cellular calcium homeostasis in chemotherapy-evoked painful peripheral neuropathy. Anesth Analg 2006; 102: 1485-90.

[22] Pastorino JG, Snyder JW, Serroni A, et al. Cyclosporin and carnitine prevent the anoxic death of cultured hepatocytes by inhibiting the mitochondrial permeability transition. J Biol Chem 1993; 268: 13791-8.

[23] Jin HW, Flatters SJ, Xiao WH, et al. Prevention of paclitaxelevoked painful peripheral neuropathy by acetyl-L-carnitine: effects on axonal mitochondria, sensory nerve fiber terminal arbors, and cutaneous Langerhans cells. Exp Neurol 2008; 210: 229-37.

[24] Cavaletti G, Gilardini A, Canta A, et al. Bortezomib-induced peripheral neurotoxicity: a neurophysiological and pathological study in the rat. Exp Neurol 2007; 204: 317-25.

[25] Broyl A, Corthals SL, Jongen JL, et al. Mechanisms of peripheral neuropathy associated with bortezomib and vincristine in patients with newly diagnosed multiple myeloma: a prospective analysis of data from the HOVON-65/GMMG-HD4 trial. Lancet Oncol 2010; 11: $1057-65$.

[26] Landowski TH, Megli CJ, Nullmeyer KD, et al. Mitochondrialmediated disregulation of $\mathrm{Ca} 2+$ is a critical determinant of Velcade (PS-341/bortezomib) cytotoxicity in myeloma cell lines. Cancer Res 2005; 65: 3828-36.

[27] Melli G, Taiana M, Camozzi F, et al. Alpha-lipoic acid prevents mitochondrial damage and neurotoxicity in experimental chemotherapy neuropathy. Exp Neurol 2008; 214: 276-84.

[28] Muthuraman A, Jaggi AS, Singh N, et al. Ameliorative effects of amiloride and pralidoxime in chronic constriction injury and vincristine induced painful neuropathy in rats. Eur J Pharmacol 2008; 587: 104-11.

[29] Kaur G, Jaggi AS, Singh N. Exploring the potential effect of Ocimum sanctum in vincristine-induced neuropathic pain in rats. $\mathrm{J}$ Brachial Plex Peripher Nerve Inj 2010; 5: 3.

[30] Xiao W, Boroujerdi A, Bennett GJ, et al. Chemotherapy-evoked painful peripheral neuropathy: analgesic effects of gabapentin and effects on expression of the alpha-2-delta type-1 calcium channel subunit. Neuroscience 2007; 144: 714-20.
[31] Flatters SJ, Bennett GJ. Ethosuximide reverses paclitaxel- and vincristine-induced painful peripheral neuropathy. Pain 2004; 109: 150-61.

[32] Gauchan P, Andoh T, Kato A, et al. Effects of the prostaglandin E1 analog limaprost on mechanical allodynia caused by chemotherapeutic agents in mice. J Pharmacol Sci 2009; 109: 469-72.

[33] Matsumoto M, Inoue M, Hald A, et al. Inhibition of paclitaxelinduced A-fiber hypersensitization by gabapentin. J Pharmacol Exp Ther 2006; 318: 735-40.

[34] Adelsberger H, Quasthoff S, Grosskreutz J, et al. The chemotherapeutic oxaliplatin alters voltage-gated $\mathrm{Na}(+)$ channel kinetics on rat sensory neurons. Eur J Pharmacol 2000; 406: 25-32.

[35] Grolleau F, Gamelin L, Boisdron-Celle M, et al. A possible explanation for a neurotoxic effect of the anticancer agent oxaliplatin on neuronal voltage-gated sodium channels. J Neurophysiol 2001; 85: 2293-7.

[36] Webster RG, Brain KL, Wilson RH, et al. Oxaliplatin induces hyperexcitability at motor and autonomic neuromuscular junctions through effects on voltage-gated sodium channels. Br J Pharmacol 2005; 146: 1027-39.

[37] Rutkove SB. Effects of temperature on neuromuscular electrophysiology. Muscle Nerve 2001; 24: 867-82.

[38] Bouhours M, Sternberg D, Davoine CS, et al. Functional characterization and cold sensitivity of T1313A, a new mutation of the skeletal muscle sodium channel causing paramyotonia congenita in humans. J Physiol 2004; 554: 635-47.

[39] Krishnan AV, Goldstein D, Friedlander M, et al. Oxaliplatin and axonal $\mathrm{Na}+$ channel function in vivo. Clin Cancer Res 2006; 12 : 4481-4.

[40] Park SB, Lin CS, Krishnan AV, et al. Oxaliplatin-induced neurotoxicity: changes in axonal excitability precede development of neuropathy. Brain 2009; 132: 2712-23.

[41] Nieto FR, Entrena JM, Cendan CM, et al. Tetrodotoxin inhibits the development and expression of neuropathic pain induced by paclitaxel in mice. Pain 2008; 137: 520-31.

[42] Joshi SK, Mikusa JP, Hernandez G, et al. Involvement of the TTXresistant sodium channel Nav 1.8 in inflammatory and neuropathic, but not post-operative, pain states. Pain 2006; 123: 75-82.

[43] Muthuraman A, Singh N. Attenuating effect of hydroalcoholic extract of Acorus calamus in vincristine-induced painful neuropathy in rats. J Nat Med 2011; 65: 480-7.

[44] Mangiacavalli S, Corso A, De Amici M, et al. Emergent T-helper 2 profile with high interleukin-6 levels correlates with the appearance of bortezomib-induced neuropathic pain. Br J Haematol 2010; 149: 916-8.

[45] Qureshi AA, Hosoi J, Xu S, et al. Langerhans cells express inducible nitric oxide synthase and produce nitric oxide. J Invest Dermatol 1996; 107: 815-21.

[46] Deng L, Ding W, Granstein RD. Thalidomide inhibits tumor necrosis factor-alpha production and antigen presentation by Langerhans cells. J Invest Dermatol 2003; 121: 1060-5.

[47] Torii H, Yan Z, Hosoi J, et al. Expression of neurotrophic factors and neuropeptide receptors by Langerhans cells and the Langerhans cell-like cell line XS52: further support for a functional relationship between Langerhans cells and epidermal nerves. J Invest Dermatol 1997; 109: 586-91.

[48] Ledeboer A, Jekich BM, Sloane EM, et al. Intrathecal interleukin10 gene therapy attenuates paclitaxel-induced mechanical allodynia and proinflammatory cytokine expression in dorsal root ganglia in rats. Brain Behav Immun 2007; 21: 686-98.

[49] Cata JP, Weng HR, Lee BN, et al. Clinical and experimental findings in humans and animals with chemotherapy-induced peripheral neuropathy. Minerva Anestesiol 2006; 72: 151-69.

[50] Sweitzer SM, Pahl JL, DeLeo JA. Propentofylline attenuates vincristine-induced peripheral neuropathy in the rat. Neurosci Lett 2006; 400: 258-61.

[51] Boyette-Davis J, Dougherty PM. Protection against oxaliplatininduced mechanical hyperalgesia and intraepidermal nerve fiber loss by minocycline. Exp Neurol 2011; 229: 353-7.

[52] McCarthy BG, Hsieh ST, Stocks A, et al. Cutaneous innervation in sensory neuropathies: evaluation by skin biopsy. Neurology 1995; 45: 1848-55.

[53] Ochoa JL, Yarnitsky D. The triple cold syndrome. Cold hyperalgesia, cold hypoaesthesia and cold skin in peripheral nerve disease. Brain 1994; 117 (Pt 1): 185-97. 
[54] Gedlicka C, Scheithauer W, Schull B, et al. Effective treatment of oxaliplatin-induced cumulative polyneuropathy with alpha-lipoic acid. J Clin Oncol 2002; 20: 3359-61.

[55] Joseph EK,Levine JD. Comparison of oxaliplatin- and cisplatininduced painful peripheral neuropathy in the rat. J Pain 2009; 10: 534-41.

[56] Kim YS, Son JY, Kim TH, et al. Expression of transient receptor potential ankyrin 1 (TRPA1) in the rat trigeminal sensory afferents and spinal dorsal horn. J Comp Neurol 2010; 518: 687-98.

[57] Wang HT, Liu ZG, Yang W, et al. Study on mechanism of bortezomib inducing peripheral neuropathy and the reversing effect of reduced glutathione. Zhonghua Xue Ye Xue Za Zhi 2011; 32: 10711 .

[58] Nakano A, Abe M, Oda A, et al. Delayed treatment with vitamin C and N-acetyl-L-cysteine protects Schwann cells without compromising the anti-myeloma activity of bortezomib. Int $\mathrm{J}$ Hematol 2011; 93: 727-35.

[59] Wang MS, Davis AA, Culver DG, et al. Calpain inhibition protects against Taxol-induced sensory neuropathy. Brain 2004; 127: 671-9.

[60] Horvath P, Szilvassy J, Nemeth J, et al. Decreased sensory neuropeptide release in isolated bronchi of rats with cisplatin-induced neuropathy. Eur J Pharmacol 2005; 507: 247-52.

[61] Jamieson SM, Liu J, Connor B, et al. Oxaliplatin causes selective atrophy of a subpopulation of dorsal root ganglion neurons without inducing cell loss. Cancer Chemother Pharmacol 2005; 56: 391-9.

[62] Tatsushima Y, Egashira N, Kawashiri T, et al. Involvement of substance $\mathrm{P}$ in peripheral neuropathy induced by paclitaxel but not oxaliplatin. J Pharmacol Exp Ther 2011; 337: 226-35.

[63] Jaggi AS, Singh N. Therapeutic targets for the management of peripheral nerve injury-induced neuropathic pain. CNS Neurol Disord Drug Targets 2011; 10: 589-609.

[64] Geppetti P, Holzer P. Neurogenic inflammation. Boca Raton: CRC Press 1996.

[65] Julius D, Basbaum AI. Molecular mechanisms of nociception. Nature 2001; 413: 203-10.

[66] Bayliss WM. On the origin from the spinal cord of the vaso-dilator fibres of the hind-limb, and on the nature of these fibres. J Physiol 1901; 26: 173-209.

[67] Lewis ST. The nocifensor system of nerves and its reactions. BMJ 1937; 194: 431-5.

[68] Nilius B, Owsianik G, Voets T, et al. Transient receptor potential cation channels in disease. Physiol Rev 2007; 87: 165-217.

[69] Basbaum AI, Bautista DM, Scherrer G, et al. Cellular and molecular mechanisms of pain. Cell 2009; 139: 267-84.

[70] Moqrich A, Hwang SW, Earley TJ, et al. Impaired thermosensation in mice lacking TRPV3, a heat and camphor sensor in the skin. Science 2005; 307: 1468-72.

[71] McKemy DD, Neuhausser WM,Julius D. Identification of a cold receptor reveals a general role for TRP channels in thermosensation. Nature 2002; 416: 52-8.

[72] Peier AM, Reeve AJ, Andersson DA, et al. A heat-sensitive TRP channel expressed in keratinocytes. Science 2002; 296: 2046-9.

[73] Jordt SE, Bautista DM, Chuang HH, et al. Mustard oils and cannabinoids excite sensory nerve fibres through the TRP channel ANKTM1. Nature 2004; 427: 260-5.

[74] Alessandri-Haber N, Dina OA, Joseph EK, et al. Interaction of transient receptor potential vanilloid 4, integrin, and SRC tyrosine kinase in mechanical hyperalgesia. J Neurosci 2008; 28: 1046-57.

[75] Miyano K, Tang HB, Nakamura Y, et al. Paclitaxel and vinorelbine, evoked the release of substance $\mathrm{P}$ from cultured rat dorsal root ganglion cells through different PKC isoform-sensitive ion channels. Neuropharmacology 2009; 57: 25-32.

[76] Vriens J, Owsianik G, Voets T, et al. Invertebrate TRP proteins as functional models for mammalian channels. Pflugers Arch 2004; 449: 213-26.

[77] Szallasi A,Blumberg PM. Vanilloid (Capsaicin) receptors and mechanisms. Pharmacol Rev 1999; 51: 159-212.

[78] Szolcsanyi J. A pharmacological approach to elucidation of the role of different nerve fibres and receptor endings in mediation of pain. J Physiol (Paris) 1977; 73: 251-9.

[79] Caterina MJ, Schumacher MA, Tominaga M, et al. The capsaicin receptor: a heat-activated ion channel in the pain pathway. Nature 1997; 389: 816-24.

[80] Attal N, Bouhassira D, Gautron M, et al. Thermal hyperalgesia as a marker of oxaliplatin neurotoxicity: a prospective quantified sensory assessment study. Pain 2009; 144: 245-52.
[81] Anand U, Otto WR,Anand P. Sensitization of capsaicin and icilin responses in oxaliplatin treated adult rat. Mol Pain 2010; 6: 82.

[82] Ta LE, Bieber AJ, Carlton SM, et al. Transient Receptor Potential Vanilloid 1 is essential for cisplatin-induced heat hyperalgesia in mice. Mol Pain 2010; 6: 1-15.

[83] Patapoutian A, Tate S, Woolf CJ. Transient receptor potential channels: targeting pain at the source. Nat Rev Drug Discov 2009; 8: 55-68.

[84] Liedtke W, Choe Y, Marti-Renom MA, et al. Vanilloid receptorrelated osmotically activated channel (VR-OAC), a candidate vertebrate osmoreceptor. Cell 2000; 103: 525-35.

[85] Delany NS, Hurle M, Facer P, et al. Identification and characterization of a novel human vanilloid receptor-like protein, VRL-2. Physiol Genomics 2001; 4: 165-74.

[86] Wissenbach U, Bodding M, Freichel M, et al. Trp12, a novel Trp related protein from kidney. FEBS Lett 2000; 485: 127-34.

[87] Suzuki M, Watanabe Y, Oyama Y, et al. Localization of mechanosensitive channel TRPV4 in mouse skin. Neurosci Lett 2003; 353: 189-92.

[88] Guler AD, Lee H, Iida T, et al. Heat-evoked activation of the ion channel, TRPV4. J Neurosci 2002; 22: 6408-14.

[89] Grant AD, Cottrell GS, Amadesi S, et al. Protease-activated receptor 2 sensitizes the transient receptor potential vanilloid 4 ion channel to cause mechanical hyperalgesia in mice. J Physiol 2007; 578: 715-33.

[90] Strotmann R, Harteneck C, Nunnenmacher K, et al. OTRPC4, a nonselective cation channel that confers sensitivity to extracellular osmolarity. Nat Cell Biol 2000; 2: 695-702.

[91] Gao X, Wu L, O'Neil RG. Temperature-modulated diversity of TRPV4 channel gating: activation by physical stresses and phorbol ester derivatives through protein kinase C-dependent and independent pathways. J Biol Chem 2003; 278: 27129-37.

[92] Watanabe H, Vriens J, Prenen J, et al. Anandamide and arachidonic acid use epoxyeicosatrienoic acids to activate TRPV4 channels. Nature 2003; 424: 434-8.

[93] Vriens J, Watanabe H, Janssens A, et al. Cell swelling, heat, and chemical agonists use distinct pathways for the activation of the cation channel TRPV4. Proc Natl Acad Sci USA 2004; 101: 396401.

[94] Yoshida T, Inoue R, Morii T, et al. Nitric oxide activates TRP channels by cysteine S-nitrosylation. Nat Chem Biol 2006; 2: 596607.

[95] Watanabe H, Vriens J, Suh SH, et al. Heat-evoked activation of TRPV4 channels in a HEK293 cell expression system and in native mouse aorta endothelial cells. J Biol Chem 2002; 277: 47044-51.

[96] O'Neil RG,Leng L. Osmo-mechanically sensitive phosphatidylinositol signaling regulates a $\mathrm{Ca} 2+$ influx channel in renal epithelial cells. Am J Physiol 1997; 273: F120-8.

[97] Pedersen S, Lambert IH, Thoroed SM, et al. Hypotonic cell swelling induces translocation of the alpha isoform of cytosolic phospholipase A2 but not the gamma isoform in Ehrlich ascites tumor cells. Eur J Biochem 2000; 267: 5531-9.

[98] Xu F, Satoh E,Iijima T. Protein kinase C-mediated Ca2+ entry in HEK 293 cells transiently expressing human TRPV4. Br J Pharmacol 2003; 140: 413-21.

[99] Alessandri-Haber N, Dina OA, Joseph EK, et al. A transient receptor potential vanilloid 4-dependent mechanism of hyperalgesia is engaged by concerted action of inflammatory mediators. J Neurosci 2006; 26: 3864-74.

[100] Chen Y, Yang C,Wang ZJ. Proteinase-activated receptor 2 sensitizes transient receptor potential vanilloid 1 , transient receptor potential vanilloid 4, and transient receptor potential ankyrin 1 in paclitaxel-induced neuropathic pain. Neuroscience 2011; 193: 44051.

[101] Alessandri-Haber N, Dina OA, Yeh JJ, et al. Transient receptor potential vanilloid 4 is essential in chemotherapy-induced neuropathic pain in the rat. J Neurosci 2004; 24: 4444-52.

[102] Alexandre J, Batteux F, Nicco C, et al. Accumulation of hydrogen peroxide is an early and crucial step for paclitaxel-induced cancer cell death both in vitro and in vivo. Int J Cancer 2006; 119: 41-8.

[103] Materazzi S, Fusi C, Benemei S, et al. TRPA1 and TRPV4 mediate paclitaxel-induced peripheral neuropathy in mice via a glutathionesensitive mechanism. Pflugers Arch 2012; 463.

[104] Odell AF, Scott JL,Van Helden DF. Epidermal growth factor induces tyrosine phosphorylation, membrane insertion, and activation 
of transient receptor potential channel 4. J Biol Chem 2005; 280: 37974-87.

[105] Sternfeld L, Anderie I, Schmid A, et al. Identification of tyrosines in the putative regulatory site of the Ca2+ channel TRPV6. Cell Calcium 2007; 42: 91-102.

[106] Atoyan R, Shander D,Botchkareva NV. Non-neuronal expression of transient receptor potential type A1 (TRPA1) in human skin. J Invest Dermatol 2009; 129: 2312-5.

[107] Streng T, Axelsson HE, Hedlund P, et al. Distribution and function of the hydrogen sulfide-sensitive TRPA1 ion channel in rat urinary bladder. Eur Urol 2008; 53: 391-9.

[108] Gratzke C, Weinhold P, Reich O, et al. Transient receptor potential $\mathrm{A} 1$ and cannabinoid receptor activity in human normal and hyperplastic prostate: relation to nerves and interstitial cells. Eur Urol 2009; 57: 902-10.

[109] Earley S, Gonzales AL, Crnich R. Endothelium-dependent cerebral artery dilation mediated by TRPA1 and $\mathrm{Ca} 2+-$ Activated $\mathrm{K}+$ channels. Circ Res 2009; 104: 987-94.

[110] Nozawa K, Kawabata-Shoda E, Doihara H, et al. TRPA1 regulates gastrointestinal motility through serotonin release from enterochromaffin cells. Proc Natl Acad Sci USA 2009; 106: 3408-13.

[111] El Karim IA, Linden GJ, Curtis TM, et al. Human odontoblasts express functional thermo-sensitive TRP channels: implications for dentin sensitivity. Pain 2011; 152: 2211-23.

[112] El Karim IA, Linden GJ, Curtis TM, et al. Human dental pulp fibroblasts express the "cold-sensing" transient receptor potential channels TRPA1 and TRPM8. J Endod 2011; 37: 473-8.

[113] Kochukov MY, McNearney TA, Fu Y, et al. Thermosensitive TRP ion channels mediate cytosolic calcium response in human synoviocytes. Am J Physiol Cell Physiol 2006; 291: C424-32.

[114] Mukhopadhyay I, Gomes P, Aranake S, et al. Expression of functional TRPA1 receptor on human lung fibroblast and epithelial cells. J Recept Signal Transduct Res 2011; 31: 350-8.

[115] Kwan KY, Allchorne AJ, Vollrath MA, et al. TRPA1 contributes to cold, mechanical, and chemical nociception but is not essential for hair-cell transduction. Neuron 2006; 50: 277-89.

[116] Sotomayor M, Corey DP, Schulten K. In search of the hair-cell gating spring elastic properties of ankyrin and cadherin repeats. Structure 2005; 13: 669-82.

[117] Bautista DM, Jordt SE, Nikai T, et al. TRPA1 mediates the inflammatory actions of environmental irritants and proalgesic agents. Cell 2006; 124: 1269-82.

[118] Story GM, Peier AM, Reeve AJ, et al. ANKTM1, a TRP-like channel expressed in nociceptive neurons, is activated by cold temperatures. Cell 2003; 112: 819-29.

[119] Sawada Y, Hosokawa H, Matsumura K, et al. Activation of transient receptor potential ankyrin 1 by hydrogen peroxide. Eur J Neurosci 2008; 27: 1131-42.

[120] Karashima Y, Talavera K, Everaerts W, et al. TRPA1 acts as a cold sensor in vitro and in vivo. Proc Natl Acad Sci USA 2009; 106: 1273-8.

[121] Zurborg S, Yurgionas B, Jira JA, et al. Direct activation of the ion channel TRPA1 by Ca2+. Nat Neurosci 2007; 10: 277-9.

[122] Nagata K, Duggan A, Kumar G, et al. Nociceptor and hair cell transducer properties of TRPA1, a channel for pain and hearing. $\mathbf{J}$ Neurosci 2005; 25: 4052-61.

[123] Knowlton WM, Bifolck-Fisher A, Bautista DM, et al. TRPM8, but not TRPA1, is required for neural and behavioral responses to acute noxious cold temperatures and cold-mimetics in vivo. Pain 2010; 150: 340-50.

[124] del Camino D, Murphy S, Heiry M, et al. TRPA1 contributes to cold hypersensitivity. J Neurosci 2010; 30: 15165-74.

[125] Bandell M, Story GM, Hwang SW, et al. Noxious cold ion channel TRPA1 is activated by pungent compounds and bradykinin. Neuron 2004; 41: 849-57.

[126] Bautista DM, Movahed P, Hinman A, et al. Pungent products from garlic activate the sensory ion channel TRPA1. Proc Natl Acad Sci USA 2005; 102: 12248-52.

[127] Bang S, Yoo S, Yang T, et al. Nociceptive and proinflammatory effects of dimethylallyl pyrophosphate via TRPV4 activation. Br J Pharmacol 2012; 2012: 1476-5381.

[128] McNamara CR, Mandel-Brehm J, Bautista DM, et al. TRPA1 mediates formalin-induced pain. Proc Natl Acad Sci USA 2007; 104: 13525-30.
[129] Bessac BF, Sivula M, von Hehn CA, et al. Transient receptor potential ankyrin 1 antagonists block the noxious effects of toxic industrial isocyanates and tear gases. FASEB J 2009; 23: 1102-14.

[130] Taylor-Clark TE, Undem BJ. Ozone activates airway nerves via the selective stimulation of TRPA1 ion channels. J Physiol 2009; 588: 423-33.

[131] Wang YY, Chang RB, Liman ER. TRPA1 is a component of the nociceptive response to CO2. J Neurosci 2010; 30: 12958-63.

[132] Andre E, Campi B, Materazzi S, et al. Cigarette smoke-induced neurogenic inflammation is mediated by alpha,beta-unsaturated aldehydes and the TRPA1 receptor in rodents. J Clin Invest 2008; 118: 2574-82.

[133] Matta JA, Cornett PM, Miyares RL, et al. General anesthetics activate a nociceptive ion channel to enhance pain and inflammation. Proc Natl Acad Sci USA 2008; 105: 8784-9.

[134] Talavera K, Gees M, Karashima Y, et al. Nicotine activates the chemosensory cation channel TRPA1. Nat Neurosci 2009; 12: 1293-9.

[135] Miyamoto T, Dubin AE, Petrus MJ, et al. TRPV1 and TRPA1 mediate peripheral nitric oxide-induced nociception in mice. PLoS One 2009; 4: e7596.

[136] Taylor-Clark TE, Undem BJ, Macglashan DW, Jr., et al. Prostaglandin-induced activation of nociceptive neurons via direct interaction with transient receptor potential A1 (TRPA1). Mol Pharmacol 2008; 73: 274-81.

[137] Materazzi S, Nassini R, Andre E, et al. Cox-dependent fatty acid metabolites cause pain through activation of the irritant receptor TRPA1. Proc Natl Acad Sci USA 2008; 105: 12045-50.

[138] Trevisani M, Siemens J, Materazzi S, et al. 4-Hydroxynonenal, an endogenous aldehyde, causes pain and neurogenic inflammation through activation of the irritant receptor TRPA1. Proc Natl Acad Sci USA 2007; 104: 13519-24.

[139] Taylor-Clark TE, McAlexander MA, Nassenstein C, et al. Relative contributions of TRPA 1 and TRPV1 channels in the activation of vagal bronchopulmonary $\mathrm{C}$-fibres by the endogenous autacoid 4oxononenal. J Physiol 2008; 586: 3447-59.

[140] Bessac BF, Sivula M, von Hehn CA, et al. TRPA1 is a major oxidant sensor in murine airway sensory neurons. J Clin Invest 2008; 118: 1899-910.

[141] Hinman A, Chuang HH, Bautista DM, et al. TRP channel activation by reversible covalent modification. Proc Natl Acad Sci USA 2006; 103: 19564-8.

[142] Macpherson LJ, Dubin AE, Evans MJ, et al. Noxious compounds activate TRPA1 ion channels through covalent modification of cysteines. Nature 2007; 445: 541-5.

[143] Andersson DA, Gentry C, Moss S, et al. Transient receptor potential A1 is a sensory receptor for multiple products of oxidative stress. J Neurosci 2008; 28: 2485-94.

[144] Dai Y, Wang S, Tominaga M, et al. Sensitization of TRPA1 by PAR2 contributes to the sensation of inflammatory pain. J Clin Invest 2007; 117: 1979-87.

[145] Rigas B,Sun Y. Induction of oxidative stress as a mechanism of action of chemopreventive agents against cancer. Br J Cancer 2008; 98: 1157-60.

[146] Nassini R, Gees M, Harrison S, et al. Oxaliplatin elicits mechanical and cold allodynia in rodents via TRPA1 receptor. Pain 2011; 152: 1621-31.

[147] Joseph EK, Chen X, Bogen O, et al. Oxaliplatin acts on IB4positive nociceptors to induce an oxidative stress-dependent acute painful peripheral neuropathy. J Pain 2008; 9: 463-72.

[148] Ramanathan B, Jan KY, Chen CH, et al. Resistance to paclitaxel is proportional to cellular total antioxidant capacity. Cancer Res 2005; 65: 8455-60.

[149] Barriere DA, Rieusset J, Chanteranne D, et al. Paclitaxel therapy potentiates cold hyperalgesia in streptozotocin-induced diabetic rats through enhanced mitochondrial reactive oxygen species production and TRPA1 sensitization. Pain 2012; 153: 553-61.

[150] Frederick J, Buck ME, Matson DJ, et al. Increased TRPA1, TRPM8, and TRPV2 expression in dorsal root ganglia by nerve injury. Biochem Biophys Res Commun 2007; 358: 1058-64.

[151] Gauchan P, Andoh T, Kato A, et al. Involvement of increased expression of transient receptor potential melastatin 8 in oxaliplatin-induced cold allodynia in mice. Neurosci Lett 2009; 458: 93-5. 
[152] Descoeur J, Pereira V, Pizzoccaro A, et al. Oxaliplatin-induced cold hypersensitivity is due to remodelling of ion channel expression in nociceptors. EMBO Mol Med 2011; 3: 266-78.

[153] Colvin LA, Johnson PR, Mitchell R, et al. From bench to bedside: a case of rapid reversal of bortezomib-induced neuropathic pain by the TRPM8 activator, menthol. J Clin Oncol 2008; 26: 4519-20.

[154] Storey DJ, Colvin LA, Mackean MJ, et al. Reversal of doselimiting carboplatin-induced peripheral neuropathy with TRPM8 activator, menthol, enables further effective chemotherapy delivery. J Pain Symptom Manage 2010; 39: e2-4.

[155] Denk F, McMahon SB. Chronic pain: emerging evidence for the involvement of epigenetics. Neuron 2012; 73: 435-44.

[156] Nassini R, Pedretti P, Moretto N, et al. Transient potential ankyrin 1 channel localized to non-neuronal airway cells promotes nonneurogenic inflammation. PLoS One 2012; 7(8): e42454.

(C) Nassini; Licensee Bentham Open.

This is an open access article licensed under the terms of the Creative Commons Attribution Non-Commercial License (http://creativecommons.org/licenses/by-nc/3.0/) which permits unrestricted, non-commercial use, distribution and reproduction in any medium, provided the work is properly cited. 